\title{
Effectiveness and safety of aspirin combined with letrozole in the treatment of polycystic ovary syndrome: a systematic review and meta-analysis
}

\author{
Qinjian Yu, Zhihui Wang, Fangfang Su, Mianmian Wang \\ Department of Obstetrics and Gynecology, The Dingli Clinical College of Wenzhou Medical University, Wenzhou, China \\ Contributions: (I) Conception and design: Q Yu, M Wang; (II) Administrative support: M Wang; (III) Provision of study materials or patients: Q Yu, \\ Z Wang, F Su; (IV) Collection and assembly of data: All authors; (V) Data analysis and interpretation: Q Yu, Z Wang, F Su; (VI) Manuscript writing: \\ All authors; (VII) Final approval of manuscript: All authors. \\ Correspondence to: Mianmian Wang. Department of Obstetrics and Gynecology, The Dingli Clinical College of Wenzhou Medical University \\ (Wenzhou Central Hospital), No.252 Baili East Road, Lucheng District, Wenzhou, China. Email: yqj19800120@163.com.
}

\begin{abstract}
Backgrounda Meta-analysis was used to evaluate the efficacy and safety of aspirin combined with letrozole in the treatment of polycystic ovary syndrome (PCOS).

Methods: Through comprehensive searches of the China Knowledge Network (CNKI), the VIP database (VIP), the Wanfang database, the China Biomedical Database (CBM), PubMed, EMBASE, and the Cochrane Library, the clinical randomized controlled trials (RCTs) published on aspirin combined with letrozole in the treatment of PCOS were collected. According to the inclusion and exclusion criteria, the included studies were screened and quality evaluated, and RevMan 5.3 software was used for meta-analysis.

Results: A total of 10 RCTs and 948 patients with PCOS were included. Meta-analysis results showed that compared with letrozole monotherapy, aspirin combined with letrozole could significantly increase the thickness of the endometrium $[M D=1.98,95 \% \mathrm{CI}: 1.63-2.34, \mathrm{P}<0.00001]$, cervical mucus scores $(\mathrm{MD}=1.65,95 \% \mathrm{CI}: 1.32-1.98, \mathrm{P}<0.00001)$, the ovulation rate $(\mathrm{OR}=3.50,95 \% \mathrm{CI}: 2.08-5.91, \mathrm{P}<0.00001)$, the number of mature follicles $(\mathrm{MD}=0.65,95 \% \mathrm{CI}: 0.51-0.78, \mathrm{P}<0.00001)$, and the pregnancy rate $(\mathrm{OR}=3.06$, 95\% CI: 2.28-4.12, $\mathrm{P}<0.00001)$, and significantly reduced the abortion rate (OR=0.20, 95\% CI: $0.11-0.38$, $\mathrm{P}<0.00001)$. There was no statistically significant difference in the incidence of adverse reactions between the 2 groups (OR=0.76, 95\% CI: 0.44-1.32, $\mathrm{P}=0.33$ ).

Conclusions: Aspirin combined with letrozole in the treatment of PCOS is safe and effective. Due to the limitations in the number and quality of the included studies, further verification with multi-center, large-sample, high-quality RCTs is still needed.
\end{abstract}

Keywords: Aspirin; letrozole; polycystic ovary syndrome (PCOS); meta-analysis

Submitted Feb 05, 2021. Accepted for publication Apr 17, 2021.

doi: 10.21037/apm-21-606

View this article at: http://dx.doi.org/10.21037/apm-21-606

\section{Introduction}

Polycystic ovary syndrome (PCOS) is believed to be a common manifestation caused by multiple etiologies, which may be the result of the combined action of mental, psychological, environmental and drug factors. PCOS is a medical condition characterized by severe hormonal imbalance, abnormal follicular development, and thickened envelopes, leading to obstruction of follicular discharge which subsequently results in infertility (1) and even more negative impacts (e.g., teratogenesis, premature delivery, miscarriage, pregnancy-related complications) (2). Knowledge about the management of PCOS is evolving. However, it remains difficult to treat PCOS largely due to the complex etiology and heterogeneous clinical manifestations. 
Therefore, symptomatic therapy is mainly used. If PCOS patients are not adequately treated for a long period of time, both the reproductive system and other systems will be affected. Letrozole, a highly effective and selective aromatase inhibitor, is able to prevent the conversion of androgens into estrogen by effectively inhibiting aromatase activity. When the level of estrogen decreases, the ability to negatively inhibit the hypothalamus-pituitary-ovarian (HPO) axis is lost. Consequently, gonadotropin is elevated for better follicle development (3). When it comes to therapeutic agents, neither the effective rate nor the pregnancy success rate of the single use of letrozole for the treatment of PCOS combined with infertility is optimistic (4). However, it is promising that some clinical studies have achieved certain success in the treatment of PCOS with aspirin combined with letrozole, but there are only a relatively small number of single-center studies and the results of clinical reports are not consistent with each other. Therefore, this systematic review and meta-analysis aims to evaluate the efficacy and safety of aspirin combined with letrozole for PCOS and infertility, providing evidence for clinical treatment.

We present the following article in accordance with the PRISMA reporting checklist (available at http://dx.doi. org/10.21037/apm-21-606).

\section{Methods}

\section{Criteria for inclusion and exclusion}

\section{Study type}

We included randomized controlled trials (RCTs) with or without blinding. Studies were restricted to those published in Chinese and English.

\section{Subjects}

We reviewed studies with participants who met the diagnostic criteria of PCOS (5), with restrictions on age, gender, and course of disease.

\section{Intervention}

The experimental group was treated with aspirin combined with letrozole, and the control group was treated with letrozole alone, with no restrictions on the total daily dose and course of treatment.

\section{Outcome measures}

(I) Endometrial thickness; (II) cervical mucus score; (III) ovulation rate; (IV) mature follicles; (V) pregnancy rate; (VI) abortion rate; (VII) adverse events (AEs).

\section{Exclusion criteria}

(I) Non-RCT study; (II) studies published in non-Chinese and non-English languages; (III) duplicate studies, experience summaries, case studies, reviews, studies with incomplete outcome data, animal experiments, meeting minutes; (IV) studies evaluating less than 15 cases; (V) patients failed to take medicine regularly and quantitatively as prescribed by the doctor; (VI) the loss rate of the subjects was $>20 \%$.

\section{Search strategy}

We searched studies through the China National Knowledge Internet (CNKI), VIP database (VIP), the Wanfang database, the Chinese Biomedical Database (CBM), MEDLINE (PubMed), EMBASE, and the Cochrane Library from inception to December 26, 2020 using Chinese and English search terms relevant to this review. The Chinese search terms used were aspirin, letrozole, polycystic ovary syndrome, and PCOS. The English search terms used were aspirin, letrozole, and polycystic ovary syndrome.

\section{Data extraction and quality assessment}

Two of the authors independently extracted general information (e.g., the first author, year of publication, research period, the number of cases, age, intervention and treatment), and outcome indicators (endometrial thickness, cervical mucus score, ovulation rate, mature follicles, pregnancy rate, miscarriage rate, adverse events). Disagreement was resolved by a third investigator. When relevant data was missed or unclear, we tried to contact the original author for accurate data, and we excluded studies without data available.

Two reviewers independently appraised studies in the search strategy for the inclusion and exclusion criteria. In case of disagreements, a third reviewer was consulted to resolve the discrepancies. The overall quality of studies included was evaluated by the improved Jadad scoring (6). The scoring includes 4 aspects: (I) generation of random sequences; (II) allocation concealment; (III) blinding method; (IV) follow up (withdrawal and quitting). Low quality studies: $1-3$ points; high quality studies: $4-7$ points.

\section{Data analysis}

The RevMan software package was used for meta-analyses 


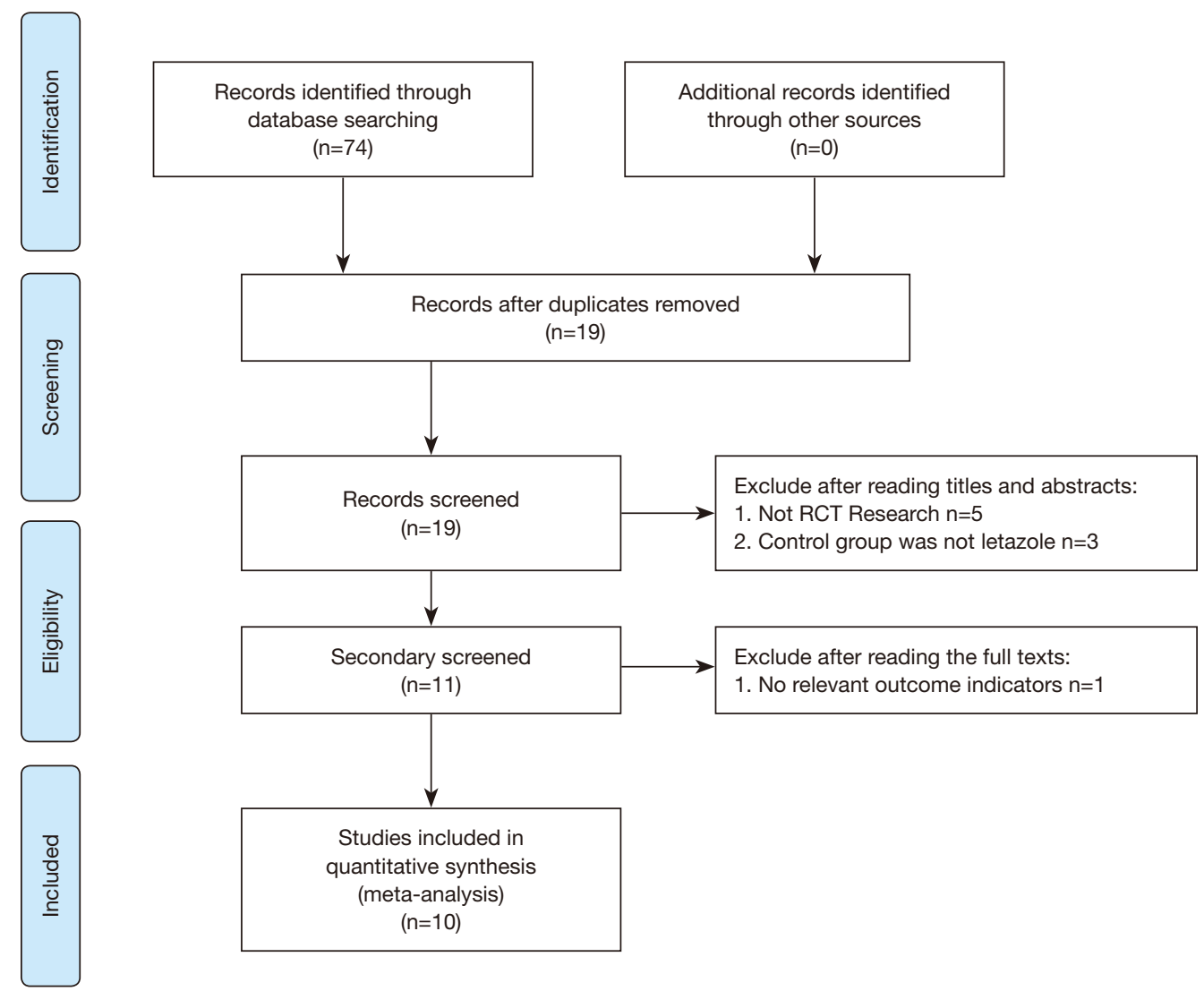

Figure 1 Study selection.

and statistical tests. The mean difference (MD) was used for measurement of continuous variables. Odds ratio (OR) was used as the effect analysis statistic, and interval estimation was presented with $95 \%$ confidence intervals (CI). The assessment of heterogeneity between the included studies was performed using the $\chi^{2}$ test. When there was no significant heterogeneity between the results $\left(\mathrm{P}>0.10, \mathrm{I}^{2} \leq 50 \%\right)$, the fixed effects model was used for meta-analysis; otherwise, the random effects model was used. Repeated operation analysis was performed to estimate the same index. One study was deleted each time to show the impact of a particular study on the combined effect, and then the study was excluded for sensitivity analysis. At the same time, the heterogeneity between subgroups was assessed when there was heterogeneity. $\mathrm{P}$ value $<0.05$ was considered statistically significant.

\section{Results}

\section{Characteristics of articles}

With the first search, we included 74 studies. Following the selection of studies according to the inclusion and exclusion criteria, 10 studies were eligible for final analysis (7-16). A total of 948 patients were included, of which 479 were in the experimental group and 469 were in the control group. The whole study selection process is depicted in Figure 1. The basic information of studies is shown in Table 1.

\section{Methodological quality evaluation results}

A total of 10 included randomized controlled articles were scored according to the modified Jadad scale (5 scored 3 marks and 5 scored 2 marks) (Table 2).

\section{Meta-analysis report}

\section{Endometrial thickness}

An improvement in endometrial thickness in patients with PCOS treated with aspirin combined with letrozole was reported in 5 studies (420 patients) $(7,9,13,15,16)$. No significant heterogeneity was found among studies $(\mathrm{P}=0.44$, $\left.\mathrm{I}^{2}=0 \%\right)$. The fixed effect model combined effect size was 
Table 1 General information of the included studies

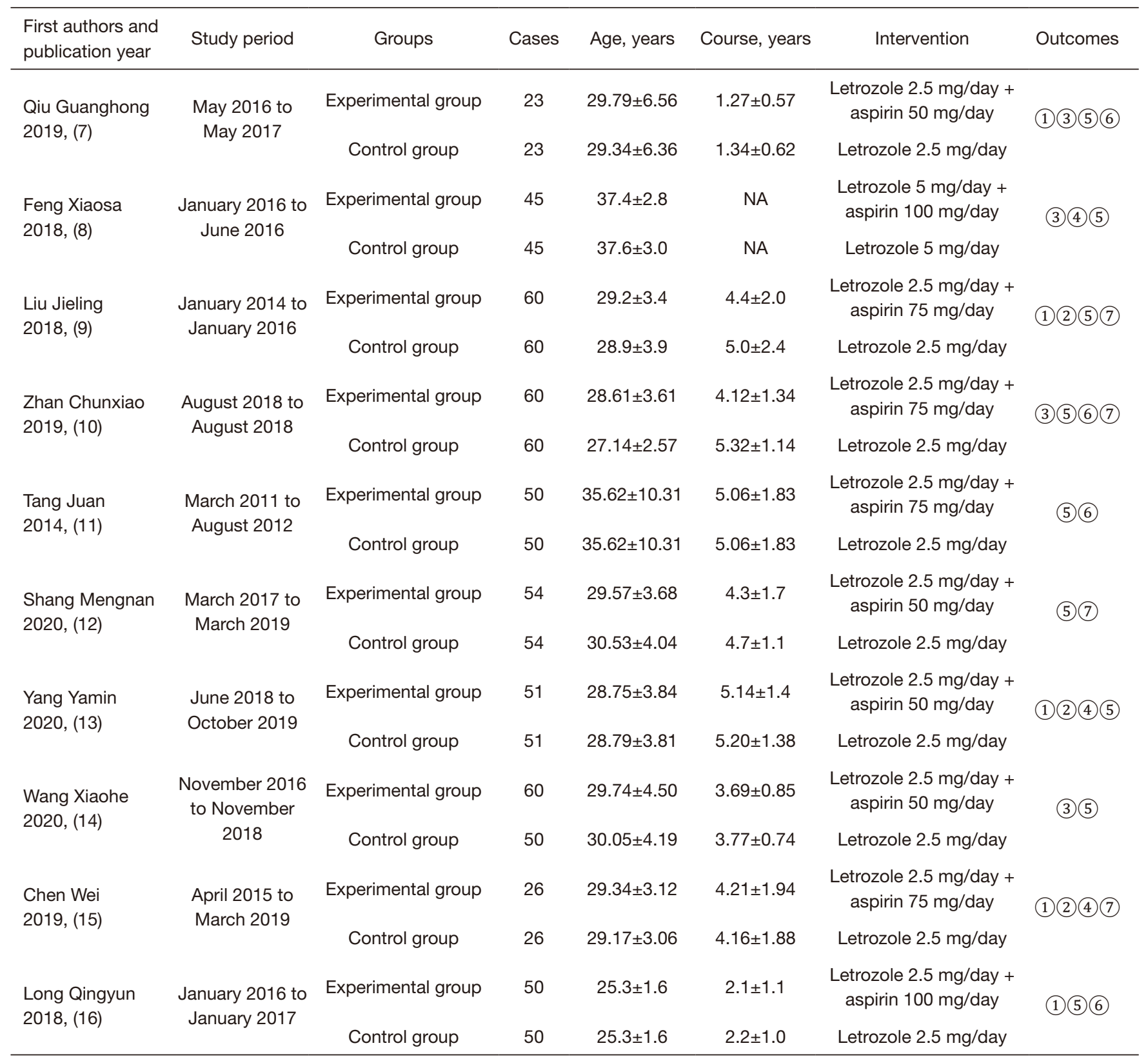

(1) endometrial thickness; (2) cervical mucus score; (3) ovulation rate; (4) mature follicles; (5) pregnancy rate; (6) abortion rate; (7) adverse events. NA, not mentioned in the paper.

used for analysis. With aspirin combined with letrozole treatment, endometrial thickness was significantly higher than that of the single use letrozole group ( $M D=1.98,95 \%$ CI: 1.63-2.34, $\mathrm{P}<0.00001$ ) (Figure 2).

\section{Cervical mucus score}

The cervical mucus score in patients with PCOS treated with aspirin combined with letrozole was assessed in 3 studies (274 patients) $(9,13,16)$. No significant heterogeneity was found among studies $\left(\mathrm{P}=0.60, \mathrm{I}^{2}=0 \%\right)$. The fixed effect model combined effect size was used for analysis. With aspirin combined with letrozole treatment, the cervical mucus score was significantly higher than that of the single use letrozole group (MD=1.65, 95\% CI: 1.32 
Table 2 Methodological quality evaluations of the included studies

\begin{tabular}{lcccc}
\hline First author and year of publication & Random methods & Allocation concealment & Blinding & Follow-up \\
\hline Guanghong Qiu, 2019, (7) & 2 & 0 & 0 & 1 \\
Xiaosa Feng, 2018, (8) & 1 & 0 & 0 & 1 \\
Jieling Liu, 2018, (9) & 1 & 0 & 0 & 1 \\
Chunxiao Zhan, 2019, (10) & 2 & 0 & 0 & 1 \\
Juan Tang, 2014, (11) & 1 & 0 & 0 & 1 \\
Mengnan Shang, 2020, (12) & 1 & 0 & 0 & 1 \\
Yamin Yang, 2020, (13) & 1 & 0 & 0 & 1 \\
Xiaohe Wang, 2020, (14) & 2 & 0 & 0 & 1 \\
Wei Chen, 2019, (15) & 2 & 0 & 0 & 3 \\
Qingyun Long, 2018, (16) & 2 & 0 & 3 \\
\hline
\end{tabular}

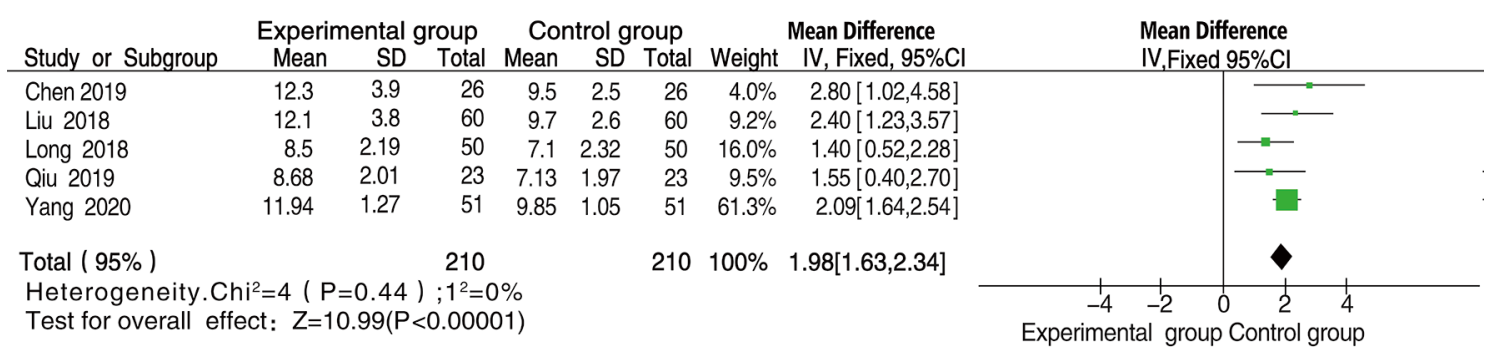

Figure 2 Forest plot of meta-analysis of the thickness of the endometrium in the 2 groups.

\subsection{8, $\mathrm{P}<0.00001$ ) (Figure 3).}

\section{Ovulation rate}

The ovulation rate in patients with PCOS treated with aspirin combined with letrozole was assessed in 4 studies (366 patients) $(7,8,10,14)$. No significant heterogeneity was found among studies $\left(\mathrm{P}=0.51, \mathrm{I}^{2}=0 \%\right)$. The fixed effect model combined effect size was used for analysis. With aspirin combined with letrozole treatment, the ovulation rate was significantly higher than that of the single use letrozole group $(\mathrm{OR}=3.50,95 \% \mathrm{CI}: 2.08-5.91, \mathrm{P}<0.00001)$ (Figure 4).

\section{Mature follicles}

Mature follicles in patients with PCOS treated with aspirin combined with letrozole were assessed in 3 studies (244 patients) $(8,13,15)$. No significant heterogeneity was found among studies $\left(\mathrm{P}=0.41, \mathrm{I}^{2}=0 \%\right)$. The fixed effect model combined effect size was used for analysis. With aspirin combined with letrozole treatment, mature follicles were significantly higher than those of the single use letrozole group ( $M D=0.65,95 \%$ CI: 0.51-0.78, $\mathrm{P}<0.00001$ ) (Figure 5).

\section{Pregnancy rate}

The pregnancy rate in patients with PCOS treated with aspirin combined with letrozole was assessed in 9 studies (896 patients) $(7-14,16)$. No significant heterogeneity was found among studies $\left(\mathrm{P}=0.86, \mathrm{I}^{2}=0 \%\right)$. The fixed effect model combined effect size was used for analysis. With aspirin combined with letrozole treatment, the pregnancy rate was significantly higher than that of the single use letrozole group (OR=3.06, 95\% CI: 2.28-4.12, $\mathrm{P}<0.00001)$ (Figure 6).

\section{Abortion rate}

The abortion rate in patients with PCOS treated with aspirin combined with letrozole was assessed in 4 studies (366 patients) $(7,10,11,16)$. No significant heterogeneity was found among studies $\left(\mathrm{P}=0.43, \mathrm{I}^{2}=0 \%\right)$. The fixed effect 


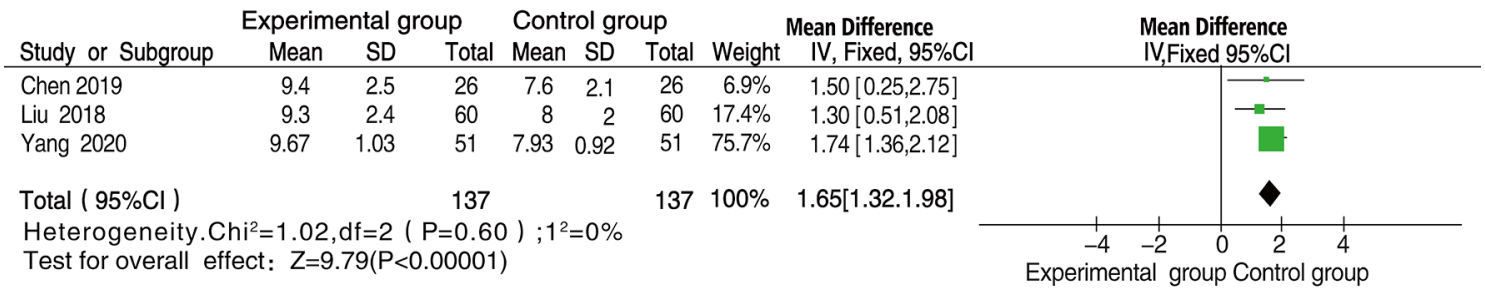

Figure 3 Forest plot of meta-analysis of the cervical mucus score in the 2 groups.

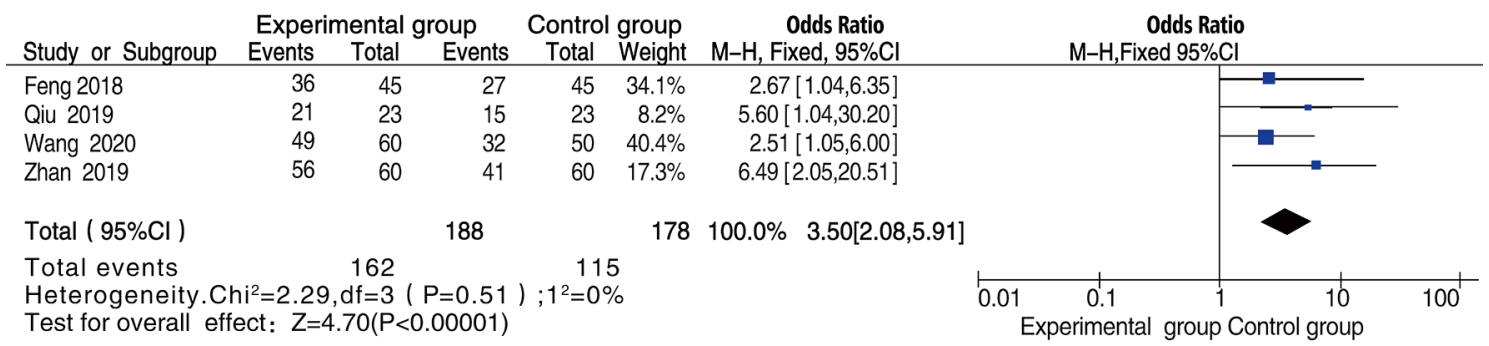

Figure 4 Forest plot of meta-analysis of the ovulation rate in the 2 groups.

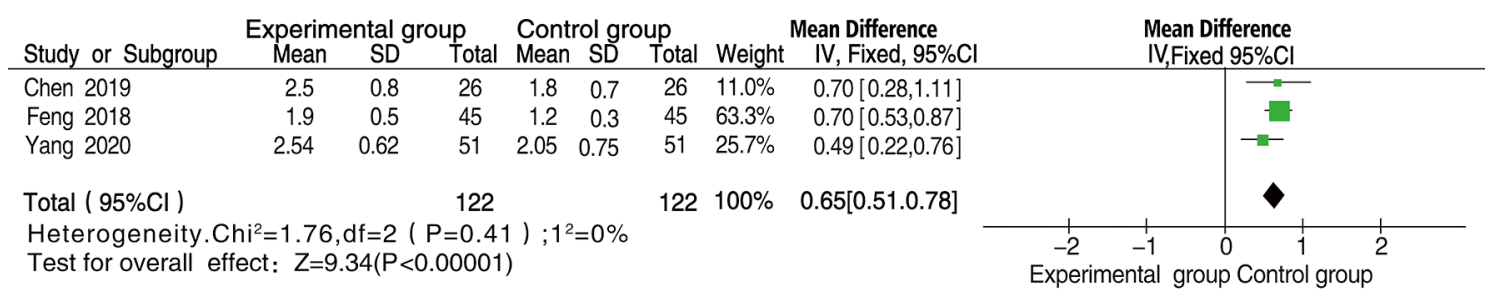

Figure 5 Forest plot of meta-analysis of the number of mature follicles in the 2 groups.

model combined effect size was used for analysis. With aspirin combined with letrozole treatment, the abortion rate was significantly lower than that of the single use letrozole group (OR=0.20, 95\% CI: 0.11-0.38, $\mathrm{P}<0.00001)$ (Figure 7).

\section{AEs}

The AEs in patients with PCOS treated with aspirin combined with letrozole were assessed in 4 studies (400 patients) $(9,10,12,15)$. No significant heterogeneity was found among studies $\left(\mathrm{P}=0.23, \mathrm{I}^{2}=30 \%\right)$. The fixed effect model combined effect size was used for analysis. There was no statistically significant difference in the incidence of AEs between the 2 groups (OR=0.76, 95\% CI: 0.44-1.32, $\mathrm{P}=0.33$ ) (Figure 8).

\section{Publication bias}

Publication bias analysis was carried out based on the pregnancy rate index (Figure 9). The symmetry of the skewed funnel plot indicates a publication bias.

\section{Discussion}

PCOS is one of the most common endocrine and metabolic disorders, accounting for $30-60 \%$ of infertility in women of child-bearing age. It is characterized by menstrual disorders or amenorrhea, hirsutism, infertility, acne, and polycystic changes in the bilateral ovaries (17). Current studies have focused on PCOS in the treatment of infertility since clinical manifestations of this disease are extremely heterogeneous. PCOS is associated with high androgen, high estrogen, and low progesterone levels in the body, leading to infertility. Letrozole is an effective aromatase inhibitor that significantly reduces the negative feedback regulation caused by estrogen on the hypothalamic 


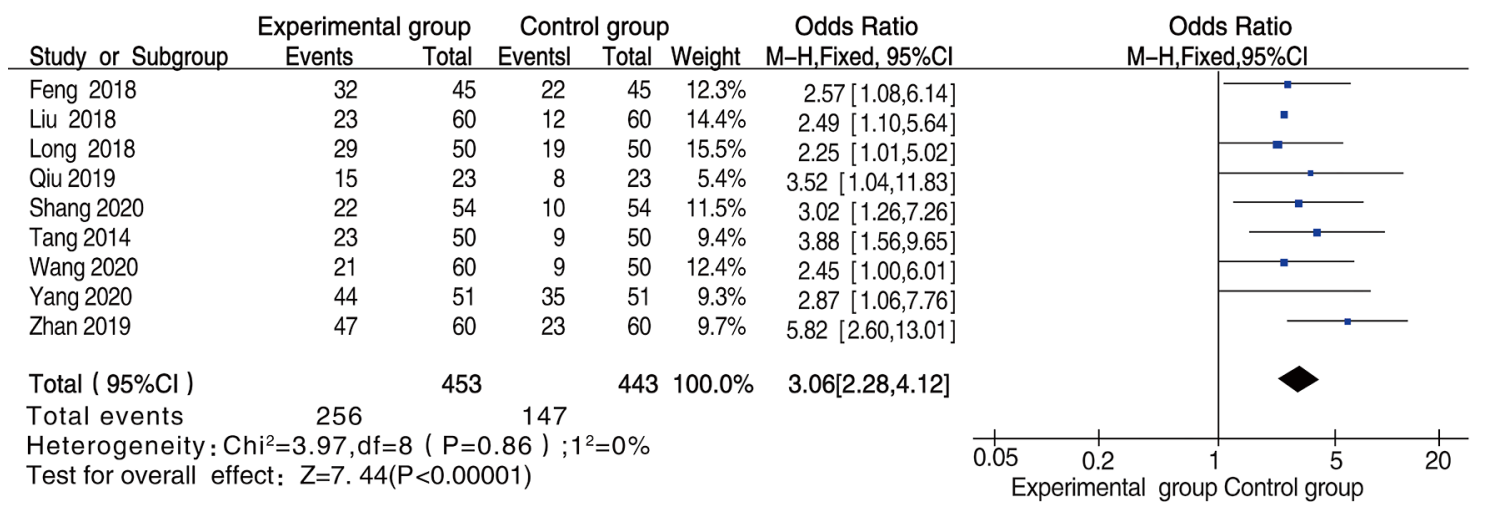

Figure 6 Forest plot of meta-analysis of the pregnancy rate in the 2 groups.

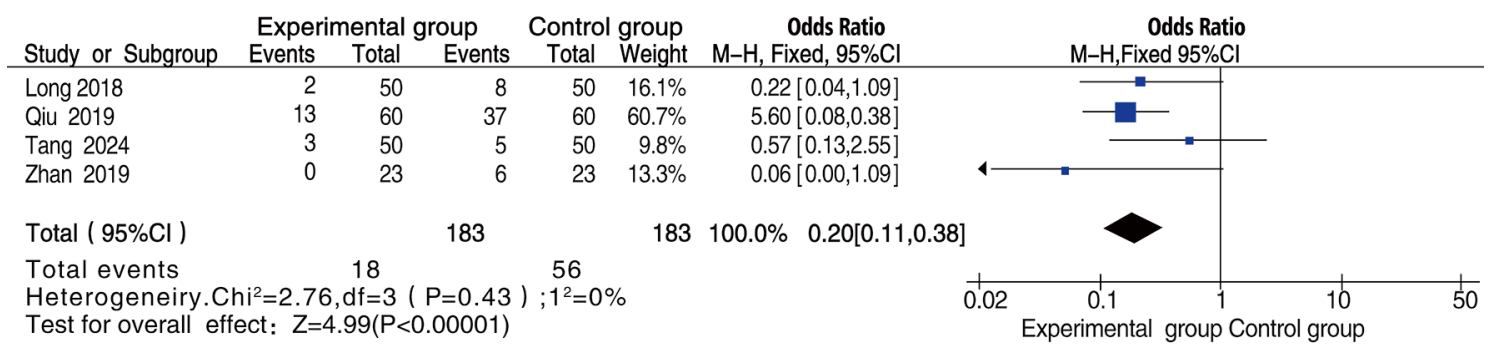

Figure 7 Forest plot of meta-analysis of the abortion rate in the 2 groups.

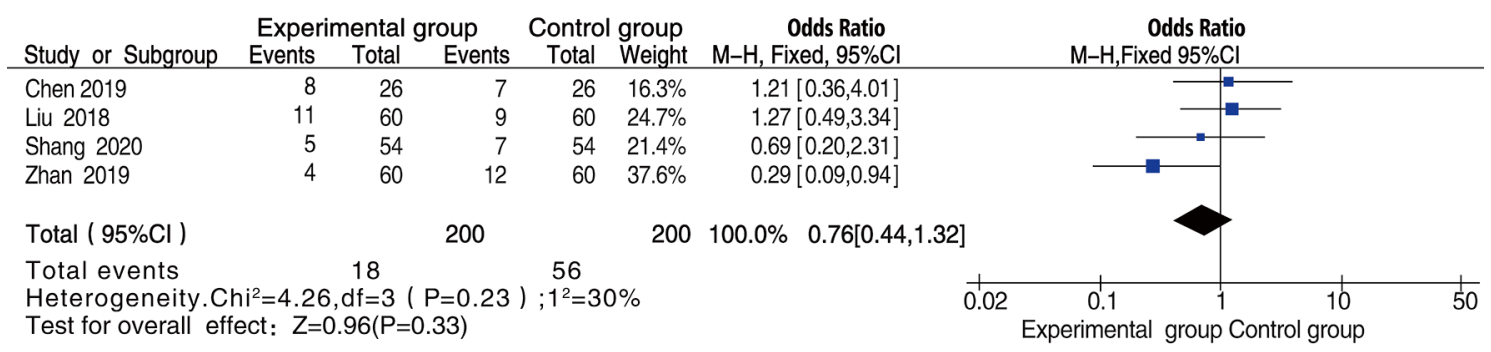

Figure 8 Forest plot of meta-analysis of the adverse reactions in the 2 groups.

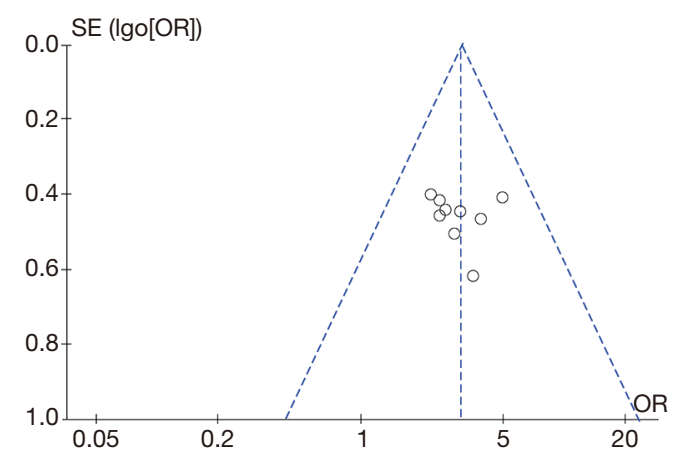

Figure 9 Funnel plot of pregnancy rate. pituitary, stimulates follicular development, promotes the ovulation cycle, improves the responsiveness of the ovaries, restores the ovulation function of the ovaries, and has a good ovulation-stimulating effect $(18,19)$. In addition, it improves the thickness of the endometrium (20,21). A previous study (22) indicated that letrozole could promote the secretion of cervical mucus for successful travel of sperm and could improve the endometrium to create a suitable environment for embryo implantation. However, the single use of letrozole for infertility of PCOS cannot effectively improve the pregnancy rate $(4,23)$. 
This meta-analysis of 10 RCTs and 948 patients with PCOS included 479 cases in the test group and 469 cases in the control group. Compared with the single use of letrozole, aspirin combined with letrozole can significantly increase endometrial thickness ( $M D=1.98,95 \%$ CI: $1.63-$ 2.34, $\mathrm{P}<0.00001)$, cervical mucus scores $(\mathrm{MD}=1.65,95 \%$ CI: $1.32-1.98, \mathrm{P}<0.00001)$, the ovulation rate $(\mathrm{OR}=3.50$, 95\% CI: $2.08-5.91, \mathrm{P}<0.00001)$, the number of mature follicles $(\mathrm{MD}=0.65,95 \% \mathrm{CI}: 0.51-0.78, \mathrm{P}<0.00001)$, and the pregnancy rate $(\mathrm{OR}=3.06,95 \% \mathrm{CI}: 2.28-4.12$, $\mathrm{P}<0.00001)$, and can significantly decrease the abortion rate $(\mathrm{OR}=0.20,95 \% \mathrm{CI}: 0.11-0.38, \mathrm{P}<0.00001)$. The hemodynamics of the reproductive organs are closely related to the functional status. Specifically, both blood perfusion and blood circulation are key factors that decide whether follicles develop normally or not. Aspirin has antiplatelet and anticoagulant effects, and is widely used in the clinical treatment of rheumatism. It also has antiinflammatory, conventional antipyretic, and analgesic effects $(24,25)$. In the treatment of PCOS, aspirin combined with letrozole achieves better therapeutic effects than letrozole alone. There are several possible explanations for the increased pregnancy rate with this combination: (I) aspirin promotes uterine artery blood flow that improves local tissue microcirculation in patients with PCOS. Additionally, it promotes endometrial development that creates better conditions for fertilized eggs to implant (26); (II) aspirin prevents platelet aggregation, promotes blood circulation in the uterus and ovaries of patients with PCOS, improves the disordered endocrine state, increases the content and concentration of progesterone, and increases the ovulation rate. Additionally, it reduces the excitability of the uterus and the sensitivity of the uterine muscle, protects the embryo, and improves the successful pregnancy rate (27); (III) aspirin significantly improves the morphology and thickness of the endometrium and increases the blood perfusion of local tissues. When combined with letrozole, aspirin maintains the balance of sex hormones, blocks the hypercoagulable reaction in the choriodecidual space, and reduces the abortion rate significantly $(28,29)$. Therefore, aspirin combined with letrozole has better results in the treatment of infertility.

There are several limitations of this meta-analysis: (I) as for the overall quality of the included studies, most of them had small sample sizes, and treatment periods varied significantly among them, despite strict standards of inclusion and exclusion criteria; (II) as for the details of the study design, RCTs were not standardized enough. For example, some studies ignored the importance of allocation concealment and blinding methods in randomized controlled studies, and some studies failed to mention the specific randomization methods; (III) the follow-up time was different in various studies, and some studies did not report the follow-up time, while the follow-up time of some studies still needed to be extended. The issues mentioned above could have affected the accuracy of the meta-analysis.

In conclusion, the combination of aspirin and letrozole is safe and effective in the treatment of infertility in PCOS. Nevertheless, a large number of multi-center and high quality RCTs are still needed for verification to overcome the limitations of the studies included.

\section{Acknowledgments}

Funding: None.

\section{Footnote}

Reporting Checklist: The authors have completed the PRISMA reporting checklist. Available at http://dx.doi. org/10.21037/apm-21-606

Conflicts of Interest: All authors have completed the ICMJE uniform disclosure form (available at http://dx.doi. org/10.21037/apm-21-606). The authors have no conflicts of interest to declare.

Ethical Statement: The authors are accountable for all aspects of the work in ensuring that questions related to the accuracy or integrity of any part of the work are appropriately investigated and resolved.

Open Access Statement: This is an Open Access article distributed in accordance with the Creative Commons Attribution-NonCommercial-NoDerivs 4.0 International License (CC BY-NC-ND 4.0), which permits the noncommercial replication and distribution of the article with the strict proviso that no changes or edits are made and the original work is properly cited (including links to both the formal publication through the relevant DOI and the license). See: https://creativecommons.org/licenses/by-nc-nd/4.0/.

\section{References}

1. Hu T, Xu KH. Research progress of etiological study on polycystic ovarian syndrom. Chin J Obstet Gynecol 
Pediatr 2016;12:360-3.

2. Teede H, Deeks A, Moran L. Polycystic ovary syndrome: a complex condition with psychological, reproductive and metabolic manifestations that impacts on health across the lifespan. BMC Med 2010;8:41.

3. Requena A, Herrero J, Landeras J, et al. Use of letrozole in assisted reproduction: a systematic review and metaanalysis. Hum Reprod Update 2008;14:571-82.

4. Amer S A, Smith J, Mahran A, et al. Double-blind randomized controlled trial of letrozole versus clomiphene citrate in subfertile women with polycystic ovarian syndrome. Hum Reprod 2017;32:1631-8.

5. Lizneva D, Suturina L, Walker W, et al. Criteria, prevalence, and phenotypes of polycystic ovary syndrome. Fertil Steril 2016;106:6-15.

6. Jadad AR, Moore RA, Carroll D, et al. Assessing the quality of reports of randomized clinical trial:is blinding necessary? Control Clin Trials 1996;17:1-12.

7. Qiu GH, Wu QL, Li XE. Study on the effect of aspirin on endometrial development and ovulation induction in PCOS patients. Capital Food and Medicine 2019;26:39-40.

8. Feng XS, Li J, He F. The effect of aspirin on hCG and E in patients with PCOS. China Maternal and Child Health Care 2018;33:790-2.

9. Liu JL, Lv ZH, Chen P, et al. Clinical efficacy, pregnancy and safety analysis of aspirin combined with letrozole in the treatment of polycystic ovary syndrome infertility. Chinese Journal of Health Care Medicine 2018;20:241-2.

10. Zhan CX. Influence of aspirin combined with letrozole on hormone levels and pregnancy rate in infertility patients with polycystic ovarian syndrome. Foreign Medical Sciences (Section of Medgeography) 2019;40:404-6.

11. Tang J. Study on treatment strategy of polycystic ovary syndrome with infertility. Medical Aesthetics and Cosmetology 2014;1:158-9.

12. Shang MN, Yu YX. Clinical effect of aspirin combined with letrozole on infertility of polycystic ovary syndrome. Psychological Monthly 2020;15:231.

13. Yang YM. Application of low dose aspirin in the adjuvant treatment of polycystic ovary syndrome. Clinical Practice of Integrated Traditional Chinese and Western Medicine 2020;20:90-1.

14. Wang XH, Ma Q, Qian ZY, et al. Effect of low dose aspirin combined with letrozole on serum visfatin, monocyte chemoattractant protein-1 and pregnancy rate in patients with polycystic ovary syndrome. Chinese Basic Medicine 2020;27:665-9.

15. Chen W. Clinical efficacy, pregnancy and safety of aspirin combined with letrozole in the treatment of polycystic ovary syndrome infertility. Special Health 2019;(11):83-4.

16. Long Q Y. Effect of letrozole combined with lowdose aspirin on endometrial development and ovulation induction in patients with polycystic ovary syndrome. China Maternal and Child Health Care 2018;33:1105-7. http://d.wanfangdata.com.cn/periodical/ChlQZXJpb2RpY2Fs Q0hJTmV3UzIwMjEwMzAyEg96Z2Z5YmoyMDE4MD UwNDkaCGhneDhpYXF6

17. Neven ACH, Laven J, Teede HJ, et al. A summary on polycystic ovary syndrome: diagnostic criteria, prevalence, clinical manifestations, and management according to the latest international guidelines. Semin Reprod Med 2018;36:5-12.

18. Wang D, Tan Y. Progress of drug research on polycystic ovary syndrome. Chinese Journal of Integrated Traditional and Western Medicine 2014;34:1022-4.

19. Hu YM, Wu WX, Xu YL. Comparative analysis of different ovulation induction regimens on pregnancy outcome of infertility patients with high luteinizing hormone (LH) polycystic ovary syndrome (PCOS). Chinese Journal of Birth Health \& Heredity 2018;26:123-6.

20. Mehdinejadiani S, Amidi F, Mehdizadeh M, et al. Effects of letrozole and clomiphene citrate on Wnt signaling pathway in endometrium of polycystic ovarian syndrome and healthy women. Biol Reprod 2019;100:641-8.

21. Gong Y, Zeng JZ, Sun Y, et al. Comparison of effects of letrozole and clomiphene citrate on ovulation induction in the infertile women with polycystic ovary syndrome. Practical Journal of Clinical Medicine 2013;10:104-7.

22. Troisi J, Cinque C, Giugliano L, et al. Metabolomic change due to combined treatment with myo-inositol, D-chiro-inositol and glucomannan in polycystic ovarian syndrome patients: a pilot study. Journal of Ovarian Research 2019;12:25.

23. Gan WJ, Li G. Research progress of ovulation induction in patients with polycystic ovary syndrome. Reproduction and Contraception 2019;39:322-4.

24. Csuka ME, McCarty DJ. Aspirin and the treatment of rheumatoid arthritis. Rheum Dis Clin North Am 1989; 15:439-54.

25. Jorda A, Aldasoro M, Aldasoro C, et al. Action of low doses of aspirin in inflammation and oxidative stress induced by a $\beta 1-42$ on astrocytes in primary culture. Int J Med Sci 2020;17:834-3.

26. Yamamoto MMW, de Medeiros SF. Differential activity of the corticosteroidogenic enzymes in normal cycling 
women and women with polycystic ovary syndrome. Reviews in Endocrine and Metabolic Disorders 2019;20:3-13.

27. Chen YQ, Li LN. Clinical effect and safety of letrozole combined aspirin for treatment of ovulation failure caused by polycystic ovarian syndrome. Chinese Journal of Human Sexuality 2015;4:112-4.

28. Xu QL, Li KB, Cai ZT, et al. Effects of different ovulation induction regimens on endometrial blood flow, thickness and type. Chinese Journal of Laboratory Diagnosis 2012;16:295-8.

29. Kong W, Wen J, Chen J. Effect of two different $\mathrm{COH}$ protocols on the IVF - ET outcome of PCOS patients. Chinese Journal of Human Sexuality 2012;21:3-6.

(English Language Editor: C. Betlazar-Maseh)
Cite this article as: $\mathrm{Yu} Q$, Wang Z, Su F, Wang M. Effectiveness and safety of aspirin combined with letrozole in the treatment of polycystic ovary syndrome: a systematic review and meta-analysis. Ann Palliat Med 2021;10(4):4632-4641. doi: 10.21037/apm-21-606 